\title{
PERMANENCE IN COARSE GEOMETRY
}

\author{
ERIK GUENTNER
}

ABSTRACT. We survey permanence results for properties of coarse metric spaces.

\section{INTRODUCTION}

During the past several years coarse metric spaces have been investigated by a large number of authors, both through their connections to conjectures concerning $\mathrm{C}^{*}$-algebra $\mathrm{K}$-theory and topological rigidity and, increasingly, as a subject of their own. See, for example, the monograph [22]. In the course of this work many properties have been introduced, frequently with specific applications in mind. Permanence results for these properties are generally scattered throughout the literature; as a property is introduced and studied its permanence characteristics are developed over time.

In this survey, we shall place the permanence results themselves at the center, our modest goal being to provide a unified perspective on permanence results for properties of coarse metric spaces. We shall identify certain primitive permanence results from which others may be derived in an axiomatic way. The primitive permanence properties include Fibering, Unions and Limits; derived results include group extensions, free products (with amalgam), etc. While we shall focus almost entirely on the properties of finite asymptotic dimension, Property A and coarse embeddability, our results apply to other properties such as the metric sparsification property [9], the property of finite decomposition complexity [17], etc.

We make no claim regarding originality. Indeed, this survey is based on the work of Bell and Dranishnikov and, to a lesser extend, Dadarlat and myself. Rather, our aim is to provide the student with an essentially self-contained survey of permanence in coarse geometry. We hope the student obtains a fresh perspective on the topics presented, a perspective they would otherwise have to extract from the literature themselves. In a few instances, our results are slight generalizations of results already in the literature and in others we have chosen to not state the most general result when a particularly simple proof of a weaker result is available. Throughout we have endeavored to provide detailed references.

During the past few years I have communicated with many people on the subject of coarse metric spaces. I have benefited immensely from these discussions, and it is a pleasure to thank my coauthors M. Dadarlat, R. Tessera, N. Wright and G. Yu and others including A. Dranishnikov and J. Roe. A special thanks to R. Willett who carefully read an early version of this work and

The author was partially supported by NSF grant DMS-0349367. 
offered many helpful comments. This survey is based on lectures given at the conferences Geometric linearization of graphs and groups held at the EPF Lausanne and the Workshop on analytic properties of infinite groups held at the Univertity of Geneva. I am indebted to Goulnara Arzhantseva and Alain Valette for invitations to these meetings and for their encouragement in writing this survey.

\section{COARSE METRIC SPACES}

In this section we shall establish the basic definitions and notations for the category of coarse metric spaces. Throughout, we allow for pseudo-metric spaces - a pseudo-metric is defined by allowing the possibility that distinct points are at distance zero.

Let $X$ and $Y$ be metric spaces. A function $f: X \rightarrow Y$ is uniformly expansive if there exists a non-decreasing function $\rho:[0, \infty) \rightarrow[0, \infty)$ such that for every $x$ and $y \in X$

$$
d(f(x), f(y)) \leq \rho(d(x, y)) ;
$$

$f$ is effectively proper if there exists a non-decreasing function $\delta:[0, \infty) \rightarrow[0, \infty)$ that is proper in the sense that $\delta(t) \rightarrow \infty$ as $t \rightarrow \infty$ and such that for every $x$ and $y \in X$

$$
\delta(d(x, y)) \leq d(f(x), f(y))
$$

There are alternate characterizations of uniformly expansive and effectively proper maps in the literature, perhaps the most common of which are treated the following lemma.

2.1. Lemma. A function $\mathrm{f}: \mathrm{X} \rightarrow \mathrm{Y}$ is uniformly expansive if and only if

$$
\forall A \exists B \text { such that } \mathrm{d}(\mathrm{x}, \mathrm{y}) \leq \mathrm{A} \Rightarrow \mathrm{d}(\mathrm{f}(\mathrm{x}), \mathrm{f}(\mathrm{y})) \leq \mathrm{B} ;
$$

$\mathrm{f}$ is effectively proper if and only if

$$
\forall \mathrm{C} \exists \mathrm{D} \text { such that } \mathrm{d}(\mathrm{f}(\mathrm{x}), \mathrm{f}(\mathrm{y})) \leq \mathrm{C} \Rightarrow \mathrm{d}(\mathrm{x}, \mathrm{y}) \leq \mathrm{D} .
$$

Proof. For any function $\mathrm{f}: \mathrm{X} \rightarrow \mathrm{Y}$ we may define

$$
\begin{array}{ll}
\rho(t) & =\sup _{d(x, y) \leq t}\{d(f(x), f(y))\}, \quad \rho(t) \in[0, \infty] \\
\delta(t) & =\inf _{d(x, y) \geq t}\{d(f(x), f(y))\},
\end{array}
$$

which are non-decreasing and satisfy the inequalities (2.1) and (2.2), respectively. Assuming the conditions (2.3) and (2.4) we observe that $\rho$ is finite and $\delta$ is proper, respectively. Conversely, assuming $f$ is uniformly expansive or effectively proper, the condition (2.3) or (2.4) is readily verified.

A function which is both uniformly expansive and effectively proper is a coarse embedding. A coarse equivalence is a coarse embedding which is coarsely onto in the sense that there exists $C>0$ such that the $C$-neighborhood of $f(X)$ is all of $Y$ :

$$
\forall y \in Y \exists x \in X \text { such that } d_{Y}(f(x), y) \leq C ;
$$


when it is necessary to keep track of the constant involved, we say $f$ is $C$-coarsely onto. Metric spaces $X$ and $Y$ are coarsely equivalent if there is a coarse equivalence $X \rightarrow Y$; although not apparent, we shall see below that coarse equivalence is an equivalence relation.

Coarse equivalences are the isomorphisms a suitable category. Objects in the category are metric spaces. Morphisms are closeness classes of uniformly expansive maps $-\mathrm{g}: \mathrm{X} \rightarrow \mathrm{Y}$ is parallel or close to $f$ if there exists $C>0$ such that for every $x \in X$

$$
d(f(x), g(x)) \leq C
$$

when necessary we say that $f$ and $g$ are $C$-close. Closeness is an equivalence relation on the set of functions from $X$ to $Y$. Observe that the composition of uniformly expansive functions is again uniformly expansive and that the compositions of two close functions with a uniformly expansive function (on either side) are again close. Henceforth, we shall refer to objects in this coarse category as coarse metric spaces.

2.2. Lemma. A function $\mathrm{f}: \mathrm{X} \rightarrow \mathrm{Y}$ is a coarse equivalence if and only if it is uniformly expansive and there exists a uniformly expansive $\mathrm{g}: \mathrm{Y} \rightarrow \mathrm{X}$ such that the compositions $\mathrm{f} \circ \mathrm{g}$ and $\mathrm{g} \circ \mathrm{f}$ are close to the identity of $\mathrm{Y}$ and $\mathrm{X}$, respectively.

Proof. Suppose $f$ is a coarse equivalence. Assume $f$ is $\mathrm{C}$-coarsely onto, and let $\rho$ and $\delta$ be as in (2.1) and (2.2). Define $g: Y \rightarrow X$ according to

$$
g(y)=\text { some } x \in X \text { satisfying } d(f(x), y) \leq C .
$$

We must show that $g$ is uniformly expansive, and that the compositions $f \circ g$ and $g \circ f$ are close to the identity. Let

$$
\begin{aligned}
\bar{\rho}(\mathrm{t}) & =\sup \{s: \delta(s) \leq t+2 \mathrm{C}\}, \\
\overline{\mathrm{C}} & =\sup \{s: \delta(s) \leq \mathrm{C}\} ;
\end{aligned}
$$

observe that since $\delta$ is proper, both $\bar{C}$ and $\bar{\rho}(t)$ are finite. The inequalities

$$
\begin{array}{r}
\delta(d(g(x), g(y)) \leq d(f g(x), f g(y)) \leq d(x, y)+2 C \\
\delta(d(g f(x), x)) \leq d(f g f(x), f(x)) \leq C
\end{array}
$$

show, respectively, that $g$ is $\bar{\rho}$-uniformly expansive and that $g \circ f$ is $\bar{C}$-close to the identity. It is immediate that $f \circ g$ is $C$-close to the identity.

Conversely, suppose $f$ and $g$ are as in the statement. Assume $g$ is $\bar{\rho}$-uniformly expansive, and that the compositions $f \circ g$ and $g \circ f$ are $\bar{C}$-close to the identity. Then $f$ is $\bar{C}$-coarsely onto. Let

$$
\delta(t)=\sup \{s: \bar{\rho}(s) \leq t-2 \bar{C}-1\} .
$$

We leave to the reader to check that $f$ is $\delta$-effectively proper, that is, $\delta$ is non-decreasing and proper, and (2.2) is satisfied.

It follows from the lemma that coarse equivalence is indeed an equivalence relation on coarse metric spaces. Also, a coarse embedding $f: X \rightarrow Y$ is a coarse equivalence $X \rightarrow f(X)$. For this reason, coarse embeddings are commonly thought of as being injective, whereas coarsely onto maps are commonly thought of as being surjective for obvious reasons. 
Remark. Although the lemma is widely known, we have provided some detail in the proof so as to make the following observation - the assertions do not depend on the particular functions but only on the associated quantitative data. For example, if a collection of functions $f_{\gamma}$ are all $\rho$-uniformly expansive, $\delta$-effectively proper and $C$-coarsely onto then their inverses $g_{\gamma}$, defined as in (2.5), are all $\bar{\rho}$-uniformly expansive and the compositions $f_{\gamma} \circ g_{\gamma}$ and $g_{\gamma} \circ f_{\gamma}$ are all $\max \{C, \bar{C}\}$-close to the identity.

Such remarks will play an important role for us, and we shall establish a framework for working effeciently with them in the next section.

\section{COARSE METRIC FAMILIES}

In what follows, it will be convenient to work with families of metric spaces, rather than a single metric space. Following [17], a metric family is simply a collection of metric spaces $\mathcal{X}=\left\{\mathrm{X}_{\alpha}\right\}$. The individual spaces comprising $X$ are its components. The index set is part of the data of a metric family. Typically, a metric family will be a collection of subspaces of a given coarse metric space, each equipped with the subspace metric. Indeed, we shall see below that if we allow extended real-valued 'metrics' this is always the case.

Let $X=\left\{X_{\alpha}\right\}$ and $y=\left\{Y_{\beta}\right\}$ be metric families. A function of families $X \rightarrow y$ is a collection of functions $\left\{f_{\gamma}\right\}$, together with a structure map $\gamma \mapsto(\alpha(\gamma), \beta(\gamma))$ relating the various index sets such that

$$
f_{\gamma}: X_{\alpha(\gamma)} \rightarrow Y_{\beta(\gamma)}
$$

and such that each $X_{\alpha}$ is the domain of some $f_{\gamma}$. The indexing sets may be different, the same space $X_{\alpha}$ may be the domain of more than one function $f_{\gamma}$, etc. We write simply $f: X \rightarrow Y$. When composing families $\left\{f_{\gamma}\right\}$ and $\left\{g_{\gamma}\right\}$ we assume the indexing sets are the same and that for every index $\gamma$ the doman of $f_{\gamma}$ is the range of $g_{\gamma}$ in which case the composition is $\left\{f_{\gamma} \circ g_{\gamma}\right\}$.

Remark. To avoid cluttering the notation we shall continue to be deliberately vague regarding 'index sets' and 'structure maps'. To minimize confusion, we shall continue with the above notation throughout: the family $X$ is always indexed by $\alpha, y$ always by $\beta$, and the function $f$ is always indexed by $\gamma$. Other, similar notation will hopefully be clear from the context.

As a general rule, quantitative statements about metric spaces, functions, etc. are applied 'uniformly' to metric families, their morphisms, etc. Thus, a function $\left\{f_{\gamma}\right\}$ of metric families is $\rho$ uniformly expansive if this is true of each $\mathrm{f}_{\gamma}$; a similar definition applies in the case of $\delta$-effectively proper functions. A function $\left\{f_{\gamma}\right\}$ is C-coarsely onto if this is true of each $f_{\gamma}$ and if, in addition, each $Y_{\beta}$ is the range of some $f_{\gamma}$. Two functions $\left\{f_{\gamma}\right\}$ and $\left\{g_{\gamma}\right\}$ are C-close if, for each $\gamma$, the functions $f_{\gamma}$ and $g_{\gamma}$ have the same domain and are C-close. The following analog of Lemma 2.2 characterizing coarse equivalence of families holds (see the remark after Lemma 2.2).

\subsection{Lemma. Let $\mathrm{f}: X \rightarrow \mathcal{X}$. The following are equivalent:}

(1) $f$ is uniformly expansive and there exists a uniformly expansive $g: y \rightarrow X$ such that the compositions $\mathrm{f} \circ \mathrm{g}$ and $\mathrm{g} \circ \mathrm{f}$ are close to the identity of $y$ and $X$, respectively;

(2) $f$ is a coarse embedding and is coarsely onto. 
When these conditions are satisfied, $\mathrm{f}$ is a coarse equivalence of families.

We close the section by returning to the point of view according to which a metric family is a family of subspaces of a single metric space - a point of view that is convenient for reducing questions about metric families to questions about singe metric spaces, and indispensible when working in the setting of coarse spaces.

Let $X=\left\{X_{\alpha}\right\}$ be a metric family and let $\gamma \mapsto \alpha(\gamma)$ be a structure map of index sets. Define an extended real-valued metric on the disjoint union

$$
X=\sqcup_{\gamma} X_{\alpha(\gamma)}=\left\{(x, \gamma): x \in X_{\alpha(\gamma)}\right\}
$$

by the formula

$$
d\left(x_{\gamma}, y_{\gamma^{\prime}}\right)= \begin{cases}d_{\alpha(\gamma)}(x, y), & \gamma=\gamma^{\prime} \\ \infty, & \text { else, }\end{cases}
$$

where we have written $x_{\gamma}$ in place of $(x, \gamma)$ when $x \in X_{\alpha(\gamma)}$. Equipped with this extended readvalued metric $X$ is the total space of $X$ (with respect to the given structure map).

While seemingly artificial, the definition of the total space permits us to repeat an individual component of $\mathcal{X}$ a number of times in the total space, enabling effecient discussion of morphisms. Thus, let $\left\{f_{\gamma}\right\}$ be a function of families $X \rightarrow Y$ and construct the total spaces $X$ and $Y$ using the structure maps that forms part of the data of $f$. The formula

$$
f(x, \gamma)=\left(f_{\gamma}(x), \gamma\right)
$$

defines a ordinary function $f: X \rightarrow Y$. Conversely, every uniformly expansive map of total spaces arises from some function of families via this construction. The next lemma collects several elementary properties.

3.2. Lemma. Suppose $\mathrm{f}$ is constructed from $\left\{\mathrm{f}_{\gamma}\right\}$ as just described. We have

(1) $\left\{f_{\gamma}\right\}$ is $\rho$-uniformly expansive if and only if $\mathrm{f}$ is;

(2) $\left\{\mathrm{f}_{\gamma}\right\}$ is $\delta$-effectively proper if and only if $\mathrm{f}$ is;

(3) $\left\{f_{\gamma}\right\}$ is C-coarsely onto if and only if $f$ is;

Further, if $\mathrm{g}$ is similarly constructed from $\left\{\mathrm{g}_{\gamma}\right\}$ then

(4) $\left\{f_{\gamma}\right\}$ and $\left\{g_{\gamma}\right\}$ are C-close if and only if $f$ and $g$ are;

and applied to $\left\{\mathrm{f}_{\gamma} \circ \mathrm{g}_{\gamma}\right\}$ the construction gives $\mathrm{f} \circ \mathrm{g}$.

When the structure map is the identity on the index set of $\mathcal{X}$ we obtain the standard total space of $X$. The original metric spaces $X_{\alpha}$ comprising $X$ are subspaces of the standard total space, and are recovered as its components - the equivalence classes for the equivalence relation defined by $x \sim y$ precisely when $d(x, y)$ is finite. In other words,

$$
X_{\alpha} \cong\left\{x: d\left(\bar{x}_{\alpha}, x\right)<\infty\right\},
$$

where $\bar{\chi}_{\alpha}$ is an arbitrarily chosen point in $X_{\alpha}$. 
Remark. For set theoretic constructions we generally require that families are defined with respect to the same index set, in which case operations are 'componentwise'. Indeed, we have already done so when discussing compositions of maps of families. We shall introduce relevant constructions as we go, but always with this general principle in mind.

Remark. We have seen that quantitative notions are typically applied to families in a 'uniform' way. An important example is that of a bounded family - a family of metric spaces of uniformly bounded diameter.

\section{COARSE STRUCTURES}

In his monograph, Roe introduced the notion of a coarse structure [22]. A coarse structure on a set $X$ is a collection $\mathcal{E}$ of subsets of $X \times X$ that contains the diagonal, and is closed under the taking of subsets, inverses, products and finite unions:

(1) $\Delta \in \mathcal{E}$

(2) $\mathrm{E} \in \mathcal{E}$ and $\mathrm{F} \subset \mathrm{E} \Rightarrow \mathrm{F} \in \mathcal{E}$

(3) $\mathrm{E} \in \mathcal{E} \Rightarrow \mathrm{E}^{-1} \in \mathcal{E}$

(4) $\mathrm{E}, \mathrm{F} \in \mathcal{E} \Rightarrow \mathrm{E} \circ \mathrm{F} \in \mathcal{E}$

(5) $E, F \in \mathcal{E} \Rightarrow E \cup F \in \mathcal{E}$

Here, the inverse and product are defined by

$$
\begin{aligned}
E^{-1} & =\{(x, y):(y, x) \in E\}, \\
E \circ F & =\{(x, y): \exists z \in X \text { such that }(x, z) \in E \text { and }(z, y) \in F\} .
\end{aligned}
$$

The subsets in $\mathcal{E}$ are called entourages or controlled sets. A set equipped with a coarse structure is a coarse space. ${ }^{1}$ A coarse space is connected if the union of its entourages is all of $\mathrm{X} \times \mathrm{X}$. While we generally assume all coarse spaces are connected, we shall discuss an exception to this convention below.

Example. A (pseudo-)metric space admits a natural coarse structure; entourages for the metric coarse structure ${ }^{2}$ are (the subsets of) the metric tubes

$$
\{(x, y): d(x, y) \leq C\} \text {. }
$$

Coarse structures and coarse spaces enjoy a philosophical advantage over coarse metric spaces - for example, we shall see below that all left invariant bounded geometry metrics on a countable group induce the same metric coarse structure which is therefore transparently uniquely determined by the group.

On the other hand, the absence of a natural guage complicates the notion of a coarse family while it is natural to speak of sets of uniform size in different metric spaces it is not possible to do so in different coarse spaces without imposing additional structure. This motivates our definition of a coarse family as a collection of coarse spaces $X=\left\{X_{\alpha}\right\}$, each of which is a coarse subspace

\footnotetext{
${ }^{1}$ On occasion the requirement that the diagonal be an entourage is dropped from the definition of coarse space; in this case, a course space in which the diagonal is an entourage is called unital.

${ }^{2}$ Roe calls this the bounded coarse structure associated to the metric [22].
} 
of a fixed external coarse space $Z$; this means that a family of entourages $\mathcal{E}$ on $Z$ is given and, for each $\alpha$, the entourages on $X_{\alpha}$ are the intersections

$$
\left\{E \cap X_{\alpha} \times X_{\alpha}: E \in \mathcal{E}\right\}
$$

The standard total coarse space of the coarse family $X$ is the coarse space $X$ whose underlying set is the disjoint union of the $X_{\alpha}$ and in which the entourages are the disjoint unions

$$
\cup_{\alpha}\left(E \cap X_{\alpha} \times X_{\alpha}\right) \subset \cup_{\alpha}\left(X_{\alpha} \times X_{\alpha}\right) \subset X \times X
$$

as $E$ ranges over $\mathcal{E}$. It is at this point that we must broaden the notion of coarse space slightly by dropping the requirement that the union of the entourages is all of $X \times X$. A coarse space failing this requirement will be called disconnected. The (connected) components of a coarse space are the equivalence classes of the following equivalence relation: $x \sim y$ precisely when there exists an entourage containing the pair $(x, y)$. The components are coarse spaces with the subspace coarse structure. A coarse space has a single component precisely when it is connected. The coarse spaces $X_{\alpha}$ comprising the family $X$ are exactly the components of the standard total coarse space of the family. This terminology agrees with that introduced earlier in the context of metric families.

In this survey we shall restrict attention to coarse metric spaces, primarily to remove a language barrier for those unfamiliar with coarse spaces. Much of the theory we shall develop applies equally to coarse spaces, and we shall attempt to formulate our results so as to make the translation to coarse spaces as transparent as possible. Ideed, we shall sprinkle remarks throughout the text to aid the reader with this translation.

\section{PROPERTIES OF COARSE METRIC SPACES}

Our main focus is on permanence results for properties of coarse metric spaces. In this short section we shall introduce the properties we consider; finite asymptotic dimension, Property $A$ and coarse embeddability. In order to state permanence results we must work with 'uniform' or 'quantitative' versions of these properties which, as we have seen, are stated most effeciently for metric families.

5.1. Finite asymptotic dimension. A coarse metric space $X$ has asymptotic dimension at most $\mathrm{d}$ if for every $R>0$ there exists a uniformly bounded cover $\mathfrak{U}$ and a partition of $\mathfrak{U}$ into $d+1$ 'colors'

$$
\mathfrak{U}=\mathfrak{U}_{0} \cup \cdots \cup \mathfrak{U}_{\mathrm{d}}
$$

in which any two (distinct) sets of the same color are R-separated:

$$
\mathrm{U}, \mathrm{V} \in \mathfrak{U}_{\mathrm{i}} \Longrightarrow \mathrm{d}(\mathrm{U}, \mathrm{V}) \geq \mathrm{R} .
$$

A coarse metric space $X$ has finite asymptotic dimension if it has asymptotic dimension at most $\mathrm{d}$, for some $\mathrm{d}$. A metric family $X$ has finite asymptotic dimension, or asymptotic dimension at most $d$ if one, equivalently each, of its total spaces does. We express these notions by writing, for example, $X \in \mathrm{FAD}_{\mathrm{d}}$ or $\mathrm{X} \in \mathrm{FAD}$. 
Remark. A metric family $X$ has finite asymptotic dimension if and only if there exists a $\mathrm{d}$ such that the components $X$ of $X$ have asymptotic dimension at most $d$ 'uniformly' in the sense of Bell and Dranishnikov [2]. Precisely, given $R$, each component $X$ admits a cover $\mathfrak{U}(X)$ which partitions into $\mathrm{d}+1$ colors as above but with

$$
\sup \{\operatorname{diam} U: U \in \mathfrak{U}(X) \text {, all } X\}<\infty .
$$

Remark. The notion of asymptotic dimension for coarse spaces was explicitly described in the monograph [22] and is easily adapted to the setting of coarse families. The essential definition is that a subset $\mathrm{U}$ of a coarse space $\mathrm{X}$ is bounded if $\mathrm{U} \times \mathrm{U}$ is an entourage; a collection of subsets $\mathrm{U}_{i}$ of $X$ is uniformly bounded if $\cup \mathrm{U}_{i} \times \mathrm{U}_{i}$ is an entourage. In a metric coarse structure these are the usual notions. See also [4].

Finite asymptotic dimension was introduced by Gromov [15]. For an up-to-date survey of this property, its application, and a wealth of examples we recommend the survey of Bell and Dranishnikov [4].

5.2. Property A and exactness. A coarse metric space is exact if it satisfies the following partition of unity condition: for every $R>0$ and every $\varepsilon>0$ there exists a partition of unity $\left\{\varphi_{u}\right\}$ subordinate to a uniformly bounded cover $\mathfrak{U}$ such that for $x$ and $y \in X$

$$
\mathrm{d}(\mathrm{x}, \mathrm{y}) \leq \mathrm{R} \Rightarrow \sum_{\mathrm{u} \in \mathfrak{U}}\left|\phi_{\mathrm{u}}(\mathrm{x})-\phi_{\mathrm{u}}(\mathrm{y})\right|<\varepsilon
$$

A metric family is exact if one, equivalently each, of its total spaces is exact. To express that a coarse metric space or metric family is exact we write $X \in \mathrm{EX}$ or $X \in \mathrm{EX}$.

Remark. A metric family $X$ is exact precisely when its components are 'uniformly' exact in the sense that given $R$ and $\varepsilon$ each component $X$ admits a partition of unity $\left\{\phi_{\mathrm{u}}^{\mathrm{X}}\right\}$ satisfying (5.2) and subordinate to a cover $\mathfrak{U}(X)$ satisfying $(5.1){ }^{3}$

Remark. The definition of exactness is easily adapted to the general setting of coarse spaces, again using the notion of boundedness mentioned above.

Dadarlat and Guentner introduced exactness for metric spaces as a substitute for Property A, believing its definition easier to manipulate than the standard characterizations of Property $A$ [12]. Further, they proved the equivalence of exactness and Property $A$ for metric spaces of bounded geometry. ${ }^{4}$

Property $A$ itself was introduced by $\mathrm{Yu}$ in the course of his work on the Novikov conjecture [25]; he was interested in defining a property that is both easy to verify in cases of interest and that implies coarse embeddability. Recently, Willett has written an excellent survey of Property A [24].

The germ of a uniform version of Property $A$ was introduced by Bell [6]; related notions play important roles in the work of Nowak [18] and Dadarlat and Guentner [12]. The equivalence of exactness and Property A works also for families - exactness of a family is equivalent to 'uniform' Property $A$ for its components.

\footnotetext{
${ }^{3}$ In the language of [12] the components of $\mathcal{X}$ are 'equi-exact'.

${ }^{4}$ Nick Wright has explained to me how to extend this equivalence to the general case. See [24].
} 
5.3. Coarse embeddability. A coarse metric space is coarsely embeddable if it admits a coarse embedding into a Hilbert space. A metric family is coarsely embeddable if it admits a coarse embedding into the family comprised of a single Hilbert space. To express that a coarse metric space or metric family is coarsely embeddable we write $X \in \mathrm{CE}$ or $X \in \mathrm{CE}$.

Remark. A metric family is coarsely embeddable precisely when its constituent spaces are 'uniformly' coarsely embeddable. Precisely, $X \in \mathrm{CE}$ if and only if each component $X$ of $X$ admits a $\rho$-uniformly expansive and $\delta$-effectively proper coarse embedding, where $\rho$ and $\delta$ are independent of $\mathrm{X}^{5}$

Remark. If the coarse metric space is countable, we may assume the Hilbert space is separable.

Remark. In defining coarse embeddability, we may work with real or complex Hilbert spaces. Indeed, viewing a complex Hilbert space as a real Hilbert space the norm is unchanged; conversely, a real Hilbert space embeds isometrically in its complexification.

Remark. In the context of coarse embeddability, restricting to coarse metric spaces entails no loss of generality. Indeed, if a coarse space coarsely embeds in a Hilbert space, or indeed in any metric space, its coarse structure is a metric coarse structure.

\section{PRIMITIVE PERMANENCE RESUltS FOR SPACES}

Let $\mathcal{P}$ be a property of coarse metric spaces; $\mathcal{P}$ could be one of the properties described above, or another property. To express the statement that a coarse metric space $X$ has the property $\mathcal{P}$ we write $X \in \mathcal{P}$.

To apply $\mathcal{P}$ to metric families, we assume that $\mathcal{P}$ satisfies the following condition: the standard total space of the family $\mathcal{X}$ satisfies $\mathcal{P}$ if and only if every total space of $\mathcal{X}$ does. We then declare that a metric family $\mathcal{X}$ has the property $\mathcal{P}$ precisely when its total spaces do, in which case we write $X \in \mathcal{P}$. In this case we also say that the components of $\mathcal{X}$ have the property $\mathcal{P}$ uniformly. These conventions are consistent with our treatment of the properties FAD $\mathrm{d}_{\mathrm{d}}$ FAD and EX; they do not quite apply to $\mathrm{CE}$, which we shall treat separately. (See section 8.)

We shall state our primitive permanence results for families, rather than for single metric spaces. This is essentially unavoidable as 'uniform' properties already enter into the statements designed for single spaces.

Coarse Invariance. If $\mathcal{X}$ and $y$ are coarsely equivalent then $\mathcal{X} \in \mathcal{P}$ if and only if $y \in \mathcal{P}$.

Subspace Permanence. If $\mathcal{X} \leq y$ and $y \in \mathcal{P}$ then $X \in \mathcal{P}$.

Our conventions regarding subspaces involve a few wrinkles. We write $X \leq y$ precisely when every component of $\mathcal{X}$ is a subspace of some component of $\mathcal{Y}$ or, in detail, when a structure map $\alpha \mapsto \beta(\alpha)$ is given and for every index $\alpha$ we have $X_{\alpha} \subset Y_{\beta(\alpha)}$. We say $X$ is a family of subspaces of $y$. We reserve the notation $X \subseteq y$ (with underline) for the special case in which the families $X$ and $y$ have the same index set and for every index $\alpha$ we have $X_{\alpha} \subset Y_{\alpha}$. In this case we say simply that $X$ is a subspace of $y$.

\footnotetext{
${ }^{5}$ In the language of [12] the components of $\mathcal{X}$ are 'equi-uniformly embeddable'.
} 
While it is essentially obvious that our basic properties FAD $\mathrm{d}_{\mathrm{d}}$ FAD and EX satisfy subspace permanence, the following lemma allows us to treat subspace permanence and coarse invariance simultaneously.

6.1. Lemma. A property $\mathcal{P}$ is coarsely invariant and satisfies subspace permanence if and only if whenever $y \in \mathcal{P}$ and $X$ coarsely embeds in $y$ then $X \in \mathcal{P}$.

Proof. A coarse embedding $X \rightarrow y$ factors as the composition of a coarse equivalence and the inclusion of a family of subspaces. Conversely, the inclusion of a family of subspaces is a coarse embedding and, if $X$ and $\mathcal{Y}$ are coarsely equivalent then each coarsely embeds in the other.

6.2. Theorem. Our basic properties, $\mathrm{FAD}_{\mathrm{d}}, \mathrm{FAD}$ and $\mathrm{EX}$ are coarsely invariant and satisfy subspace permanence. Precisely, let $\mathcal{P}$ be one of these properties. If $\mathcal{Y} \in \mathcal{P}$ and $\mathcal{X}$ coarsely embeds in $y$ then $X \in \mathcal{P}$.

Proof. For the properties $\mathrm{FAD}_{\mathrm{d}}$, FAD and EX the result for families follows either directly or, with aid of Lemma 3.2 after passing to total spaces, from the analogous (and well-known) result for single metric spaces. See $[2,22]$ and [12, Rem. 2.11].

Our next permanence property concerns the attempt to conclude that a coarse metric space has property $\mathcal{P}$ from the knowledge that it is written as the union of subspaces each of which have property $\mathcal{P}$. Clearly the subspaces must have property $\mathcal{P}$ uniformly. Further, if not every (countable) space has property $\mathcal{P}$, an additional hypothesis, typically some sort of excision condition, is necessary in general.

Union Permanence. Suppose $z=X \cup \mathcal{X}$. If $X$ and $y \in \mathcal{P}$ then $z \in \mathcal{P}$.

Suppose $Z=\cup X_{i}$. Suppose further that for every $R>0$ there exists a subspace $\mathcal{W}=\mathcal{W}(\mathrm{R}) \subseteq z$ such that $\mathcal{W} \in \mathcal{P}$ and such that for every index $\alpha$ the collection $\left\{X_{i, \alpha}-W_{\alpha}\right\}_{i}$ is $R$-disjoint. If the $X_{i, \alpha}$ have $\mathcal{P}$ uniformly then $z \in \mathcal{P}$.

In accord with our conventions, we write $Z=\cup_{i} X_{i}$ when $Z$ and the $X_{i}$ share the same index set and, in addition, for each index $\alpha$ we have $Z_{\alpha}=\cup_{i} X_{i, \alpha}$. In particular, all families in the statement are indexed by the same set.

Remark. The assumption that the $X_{i, \alpha}$ have $\mathcal{P}$ uniformly means that the family $\left\{X_{i, \alpha}\right\}$, as both $\alpha$ and $i$ vary, satisfies $\mathcal{P}$. The $X_{i, \alpha}-W_{\alpha}$, for fixed $\alpha$ and varying $i$, are subspaces of $Z_{\alpha}$, allowing us to speak of of R-disjointness.

Remark. If the property $\mathcal{P}$ is coarsely invariant and satisfies subspace permanence then the finite assertion of union permanence follows from the infinite assertion. Indeed, excise the R-neighborhood of the intersection $X \cap Y$ from each and observe:

(1) $X-N_{R}(X \cap Y)$ and $Y-N_{R}(X \cap Y)$ are R-disjoint;

(2) $N_{R}(X \cap Y)$ is coarsely equivalent to $X \cap Y$, which is a subspace of both $X$ and $Y$.

The formulation for families is left to the reader. This observation applies to each of our basic properties. 


\subsection{Theorem. The basic properties $\mathrm{FAD}_{\mathrm{d}}$ and $\mathrm{EX}$ satisfy union permanence.}

Proof. Again, after passing to (standard) total spaces the result follows from the analogous statement for metric spaces - one merely checks that the proofs are valid in the case of extended real-valued metrics. Observe, for example, that the assertion $Z=\cup_{i} X_{i}$ translates to $Z=\cup_{i} X_{i}$ at the level of (standard) total spaces. See $[2,12]$.

While finite union permanence works without change for FAD, a little additional care must be taken to formulate the general case of union permanence. Indeed, if the conclusion $z \in$ FAD is to be true then the excised subspaces $\mathcal{W}(\mathrm{R})$ must have asymptotic dimension no greater than that of $z$. Taking this into account, we obtain a correct formulation of union permanence for FAD, which is merely a thinly disguised version of union permanence for $\mathrm{FAD}_{\mathrm{d}}$ and follows directly from it.

6.4. Theorem. Suppose $z=\cup X_{i}$. Suppose that there exists $\mathrm{d}$ such that for every $\mathrm{R}>0$ there exists a subspace $\mathcal{W}=\mathcal{W}(\mathrm{R}) \subseteq \mathcal{Z}$ such that $\mathcal{W} \in \mathrm{FAD}_{\mathrm{d}}$ and such that the collection $\left\{\mathrm{X}_{i, \alpha}-\mathrm{W}_{\alpha}\right\}_{\mathrm{i}}$ is $\mathrm{R}$-disjoint. If $\left\{\mathrm{X}_{i, \alpha}\right\} \in \mathrm{FAD}$ then $\mathrm{z} \in \mathrm{FAD}$.

Our next, and perhaps most important permanence property concerns the attempt to conclude that a coarse metric space has property $\mathcal{P}$ by fibering it, in the coarse category, over a space with property $\mathcal{P}$ in such a way that the fibers have property $\mathcal{P}$.

Fibering Permanence. Let $f: X \rightarrow y$ be uniformly expansive. Suppose $y \in \mathcal{P}$ and that for every bounded family of subspaces $z \leq y$ the inverse image $f^{-1}(z) \in \mathcal{P}$. Then $X \in \mathcal{P}$.

Before discussing the conventions relevant for the statement, we illustrate the hypotheses with a simple yet motivating example in the context of single spaces.

Example. Consider the projection $\mathrm{X}=\mathrm{X}^{\prime} \times \mathrm{Y} \rightarrow \mathrm{Y}$. In this case we also have $\mathrm{Y} \subset \mathrm{X}$, so that if the property $\mathcal{P}$ satisfies subspace permanence the portion of the hypothesis pertaining to $Y$ is necessary. The remaining hypothesis amounts to the assertion that $X^{\prime} \in \mathcal{P}$. In fact, if $\mathcal{P}$ satisfies coarse invariance, subspace and fibering permanence then a product has property $\mathcal{P}$ if and only if each of its factors do.

Our conventions regarding the inverse image are the natural ones $-f^{-1}(z) \leq X$ is the family of inverse images of the spaces comprising $z$, under the maps comprising $f$. To give a precise definition, recall that as part of the data of the family of subspaces $z \leq y$ we have a structure map $\delta \mapsto \beta(\delta)$ and, as part of the data of $f$ we have the structure map $\gamma \mapsto(\alpha(\gamma), \beta(\gamma))$. When the index pair $(\gamma, \delta)$ satisfies $\beta(\gamma)=\beta(\delta)$ we have

$$
\mathrm{Z}_{\delta} \subset \mathrm{Y}_{\beta(\delta)}, \quad \mathrm{f}_{\gamma}: \mathrm{X}_{\alpha(\gamma)} \rightarrow \mathrm{Y}_{\beta(\delta)}
$$

so that $f_{\gamma}^{-1}\left(Z_{\delta}\right) \subset X_{\alpha(\gamma)}$. We define the inverse image by

$$
f^{-1}(z)=\left\{f_{\gamma}^{-1}\left(Z_{\delta}\right): \beta(\delta)=\beta(\gamma)\right\}
$$

a family of subspaces of $\mathcal{X}$ with index set $\{(\gamma, \delta): \beta(\gamma)=\beta(\delta)\}$ and structure map $(\gamma, \delta) \mapsto$ $\alpha(\gamma)$.

6.5. Theorem. Property EX satisfies fibering permanence. 
Proof. As usual, a direct proof without appeal to total spaces is possible, and amounts to careful bookkeeping with the constants appearing in the proof for a single space. The proof is a combination of Thm. 3.1 and Cor. 3.3 of [12].

Fibering permanence is somewhat more subtle than the other permanence properties, and care must be taken to formulate it correctly for our other basic properties. For asymptotic dimension, the following result is both simple and useful.

6.6. Theorem. Let $\mathrm{f}: X \rightarrow y$ be uniformly expansive. Suppose that $y \in$ FAD. Suppose there exists $d$ such that for every bounded family of subspaces $z \leq y$ the inverse image $f^{-1}(z) \in \operatorname{FAD}_{d}$. Then $X \in$ FAD.

Remark. In the case of single spaces, as opposed to families, significantly more refined results are possible. Perhaps the first such result is [5, Thm. 1], which is stated for a Lipschitz map between geodesic metric spaces, and achieved a bound much better than that inherent in our proof. An optimal result was obtained recently in [7]. The reader may wish to adapt these to the setting of families. See the survey [4] for more details.

In the proof, and subsequently, we shall work with an obvious reformulation of finite asymptotic dimension in terms of colorings. For example, the conclusion $\mathcal{X} \in \mathrm{FAD}_{\mathrm{d}^{\prime \prime}}$ is rephrased as follows: for every $R$ there exists an $S$ such that for every $\alpha$ there exists a cover $\mathfrak{U}_{\alpha}$ of $X_{\alpha}$ and a coloring $c_{\alpha}: \mathfrak{U}_{\alpha} \rightarrow\left\{0, \ldots, d^{\prime \prime}\right\}$ satisfying

(1) if $\mathrm{U} \in \mathfrak{U}_{\alpha}$ then $\operatorname{diam}(\mathrm{U}) \leq \mathrm{S}$;

(2) if $\mathrm{U} \neq \mathrm{V} \in \mathfrak{U}_{\alpha}$ and $\mathrm{c}_{\alpha}(\mathrm{U})=\mathrm{c}_{\alpha}(\mathrm{V})$ then $\mathrm{d}(\mathrm{U}, \mathrm{V}) \geq \mathrm{R}$.

For brevity we shall express (1) by saying that $\mathfrak{U}_{\alpha}$ is $S$-bounded, and (2) by saying that $c_{\alpha}$ is a $\left(d^{\prime \prime}, R\right)$-coloring of $\mathfrak{U}_{\alpha}$.

Proof. For variety, and to illustrate the alternate 'quantitative' point of view, we shall give a direct proof without appeal to total spaces.

The statement is a thinly disguised version of the simplest type of result concerning asymptotic dimension of a product - and is proven the same way. Compare [11]. So motivated, we assume $y \in$ FAD $_{d^{\prime}}$ and shall prove that $X \in$ FAD $_{d^{\prime \prime}}$, where $d^{\prime \prime}=(d+1)\left(d^{\prime}+1\right)-1$. Further, we restrict attention to the (equivalent) case in which $f, X$ and $y$ are all defined over the same index set; thus we have a family of $\rho$-uniformly expansive maps

$$
f_{\alpha}: X_{\alpha} \rightarrow Y_{\alpha}
$$

As a final preparation we rephrase the preimage condition in the statement: for every $S^{\prime}$ and $R$ there exists an $S$ such that for every subset $U$ of every $Y_{\alpha}$ satisfying $\operatorname{diam}(U) \leq S^{\prime}$ there exists a cover $\mathfrak{W}$ of $f_{\alpha}^{-1}(U)$ with the following two properties:

(1) $\mathfrak{W}$ is S-bounded;

(2) $\mathfrak{W}$ admits a $(d, R)$-coloring.

(Note that the cover comprised of all subsets of the $Y_{\alpha}$ of diameter $\leq S^{\prime}$ is bounded.) 
We turn to the proof. Assume $f$ is $\rho$-uniformly expansive. Let $R$ be given. We apply the hypothesis $y \in$ FAD $_{d^{\prime}}$ to obtain $S^{\prime}$ and covers $\mathfrak{V}_{\alpha}$ of $Y_{\alpha}$, each of which is $S^{\prime}$-bounded and admits a $\left(d^{\prime}, \rho(R)\right)$-coloring $c_{\alpha}$. We lift these to $X_{\alpha}$ by defining

$$
\begin{gathered}
\overline{\mathfrak{V}}_{\alpha}=\left\{f_{\alpha}^{-1}(\mathrm{U}): \mathrm{U} \in \mathfrak{V}_{\alpha}\right\} \\
\overline{\mathrm{c}}_{\alpha}(\overline{\mathrm{U}})=\mathrm{c}_{\alpha}(\mathrm{U}), \quad \text { some } \mathrm{U} \in \mathfrak{V}_{\alpha} \text { for which } \overline{\mathrm{U}}=\mathrm{f}_{\alpha}^{-1}(\mathrm{U}) .
\end{gathered}
$$

(It may happen that two distinct elements of $\mathfrak{V}_{\alpha}$ have the same preimage and in order that $\overline{\mathbf{c}}_{\alpha}$ is well-defined we must choose one of them.) It follows from the fact that $f_{\alpha}$ is $\rho$-uniformly expansive that $\overline{\mathrm{c}}_{\alpha}$ is a $\left(\mathrm{d}^{\prime}, \mathrm{R}\right)$-coloring of $\overline{\mathfrak{V}}_{\alpha}$.

As the covers $\mathfrak{V}_{\alpha}$ are all $S^{\prime}$-bounded the preimage condition applies. We obtain $S$ with the property that each $\overline{\mathrm{U}}$ admits a cover $\mathfrak{W}(\overline{\mathrm{U}})$ that is $S$-bounded and admits a $(\mathrm{d}, \mathrm{R})$-coloring $\mathrm{c}_{\overline{\mathrm{U}}}$. The cover and coloring we require are defined:

$$
\begin{gathered}
\mathfrak{U}_{\alpha}=\left\{\mathrm{V} \in \mathfrak{W}(\overline{\mathrm{U}}): \text { all } \overline{\mathrm{U}} \in \overline{\mathfrak{V}}_{\alpha}\right\} \\
\mathrm{c}_{\alpha}(\mathrm{V})=\left(\overline{\mathrm{c}}_{\alpha}(\overline{\mathrm{U}}), \mathrm{c}_{\overline{\mathrm{U}}}(\mathrm{V})\right), \quad \text { some } \overline{\mathrm{U}} \text { for which } \mathrm{V} \in \mathfrak{W}(\overline{\mathrm{U}}) .
\end{gathered}
$$

(Again, we make choices so that $c_{\alpha}$ is well-defined.) This is a coloring with values in $\left\{0, \ldots, d^{\prime}\right\} \times$ $\{0, \ldots, d\}$. Each $\mathfrak{U}_{\alpha}$ is $S$-bounded. If distinct sets have the same color, they could be from a common $\mathfrak{W}(\overline{\mathrm{U}})$ - in which case they are at distance $\geq R$ because their colors agree in the second coordinate and $\mathfrak{W}(\overline{\mathrm{U}})$ is $(\mathrm{d}, \mathrm{R})$-colored - or from distinct ones $\mathfrak{W}(\overline{\mathrm{U}})$ and $\mathfrak{W}\left(\overline{\mathrm{U}}_{1}\right)$ - in which case they are at distance $\geq R$ because this is already true of $\overline{\mathrm{U}}$ and $\overline{\mathrm{U}}_{1}$ as like-colored members of the $\left(\mathrm{d}^{\prime}, \mathrm{R}\right)$-colored $\overline{\mathfrak{V}}_{\alpha}$.

Our final primitive permanence result will be useful when we disucss direct unions of discrete groups in the next section. While the properties $\mathrm{FAD}_{\mathrm{d}}$ and EX satisfy limit permanence FAD does not, for essentially obvious reasons.

Limit Permanence. Suppose that for every $R>0$ there exists a decomposition $Z=\cup_{i} X_{i}$ such that for every index $\alpha$ the collection $\left\{\mathrm{X}_{i, \alpha}\right\}$ is $\mathrm{R}$-disjoint and such that the $\mathrm{X}_{i, \alpha}$ satisfy $\mathcal{P}$ uniformly. Then $\mathrm{Z} \in \mathcal{P}$.

\subsection{Theorem. The properties $\mathrm{FAD}_{\mathrm{d}}$ and $\mathrm{EX}$ satisfy limit permanence.}

Proof. While straightforward, limit permanence is not treated explicitly elsewhere. First, for $\mathrm{FAD}_{\mathrm{d}}$, let $\mathrm{R}$ be given. Obtain a decomposition $\mathcal{Z}=\cup X_{i}$ as in the statement of limit permanence. The hypothesis now implies that there exists $S$ such that for each $X_{i, \alpha}$ there exists an $S$-bounded cover $\mathfrak{U}_{i, \alpha}$ admitting a $(d, R)$-coloring. Taken together, $\mathfrak{U}_{\alpha}=\cup_{i} \mathfrak{U}_{i, \alpha}$ is an $S$-bounded cover of $Z_{\alpha}$ admitting a $(d, R)$-coloring.

The proof for EX is no more difficult, and is omitted.

We close this section with a single derived permanence result for spaces, which holds for our basic properties FAD and EX.

6.8. Theorem. Let $\mathcal{P}$ be a property satisfying subspace and fibering permanence and for which $\mathbb{R} \in \mathcal{P}$. A metric family satisfies $\mathcal{P}$ precisely when the collection of all its bounded subspaces satisfies $\mathcal{P}$ uniformly. 
Proof. Let $\mathcal{P}$ be as in the statement, and let $\mathcal{X}$ be a metric family. The converse being immediate, we show that if the collection of all bounded subspaces of $\mathcal{X}$ satisfies $\mathcal{P}$ unifromly then $\mathcal{X}$ itself satisfies $\mathcal{P}$.

Fix a basepoint $\chi_{\alpha}$ in each component $X_{\alpha}$ of $X$. Define a contractive, in particular uniformly expansive map $f: X \rightarrow\{\mathbb{R}\}$ using the distance function:

$$
f_{\alpha}(x)=d\left(x_{\alpha}, x\right), \quad f_{\alpha}: X_{\alpha} \rightarrow \mathbb{R} .
$$

If now $Z$ is a bounded family of subspaces of $\mathbb{R}$ then $f^{-1}(Z)$ is a family of bounded subspaces of $X$, which satisfies $\mathcal{P}$ by hypothesis. Thus, fibering permanence applies.

Remark. In the proof, the distinction between 'family of bounded subspaces' and 'bounded family of subspaces' is purposeful.

The theorem asserts a form of 'locality' for $\mathcal{P}$, and may be rephrased in several suggestive ways. We work with a single space $X$. First, assuming $\mathcal{P}$ satisfies subspace permanence we may rephrase by asserting equivalence of the following:

(1) $X \in \mathcal{P}$;

(2) the family $\{B(x, R)\}$, as both $x \in X$ and $R \in \mathbb{R}$ vary, satisfies $\mathcal{P}$;

(3) for each fixed $x \in X$ the family $\{B(x, R)\}$, as $R \in \mathbb{R}$ varies, satisfies $\mathcal{P}$.

Second, for a locally finite space $X$ we have: $X \in \mathcal{P}$ precisely when the collection of its finite subsets satisfies $\mathcal{P}$ uniformly. In this form the result was known for property $\mathrm{CE}$ for quite some time [14]. More recently, a version was formulated and proved for Property A [8]; see also [24] for another variant.

\section{DERIVED PERMANENCE RESULTS FOR GROUPS}

Motivated by the wealth of applications we focus on the case of (countable) groups. The essential idea is to prove that a group $G$ has property $\mathcal{P}$ by observing that it acts by isometries on a space having property $\mathcal{P}$ in such a way that the stabilizers of the action have property $\mathcal{P}$ - an application of fibering permanence. A closely related question concerns proving that $\mathrm{G}$ has property $\mathcal{P}$ assuming it is built from groups having property $\mathcal{P}$ by familiar constructions from group theory. Henceforth, we shall work with single spaces and single groups, leaving to the reader to formulate appropriate 'family' versions.

7.1. Groups as coarse metric spaces. Let $G$ be a group. A length function on $G$ is a function $\ell: G \rightarrow[0, \infty)$ satisfying

(1) $\ell(1)=0$

(2) $\ell\left(s^{-1}\right)=\ell(s)$

(3) $\ell(s t) \leq \ell(s)+\ell(t)$

We allow for the possibility that some non-identity elements of $G$ have length zero. A length function is proper if, for every $C \geq 0$, the set

$$
\{s \in G: \ell(s) \leq C\}
$$


is finite. One easily checks that a group admits a proper length function precisely when it is countable. A metric on $\mathrm{G}$ is left invariant if it satisfies

$$
d(r s, r t)=d(s, t)
$$

for every $r, s$ and $t \in G$. If $\ell$ is a length function on $G$ then $d(s, t)=\ell\left(s^{-1} t\right)$ defines a left invariant metric on $G$; conversely, by setting $\ell(s)=d(1, s)$ one checks that every left invariant metric on $G$ arises in this way. A length function is proper if and only if the corresponding metric has bounded geometry, in the sense that there is a uniform bound on the cardinality of a ball of a fixed radius (independent of its center). We shall always assume a metric on a group to be left invariant.

7.1.1. Proposition. Let $\mathrm{G}$ be a countable group. Up to coarse equivalence, $\mathrm{G}$ admits a unique bounded geometry metric. Indeed, if $\mathrm{d}_{1}$ and $\mathrm{d}_{2}$ are bounded geometry metrics on $\mathrm{G}$ then the identity map $\left(\mathrm{G}, \mathrm{d}_{1}\right) \rightarrow\left(\mathrm{G}, \mathrm{d}_{2}\right)$ is a coarse equivalence.

When viewing a (countable) group $G$ as a metric space, the statement that $G$ possesses a particular property is always understood with reference to a bounded geometry metric. In light of the proposition, for properties satisfying coarse invariance such an assertion is unambiguous.

Example. A discrete group G (countable or not) admits a natural coarse structure; entourages for the canonical (left invariant bounded geometry) coarse structure are (the subsets of) the tubes

$$
\left\{(\mathrm{s}, \mathrm{t}): \mathrm{s}^{-1} \mathrm{t} \in \mathrm{U}, \mathrm{U} \subset \mathrm{G} \text { finite }\right\} .
$$

While this definition is approprate for uncountable discrete groups, when $\mathrm{G}$ is countable it agrees with the metric coarse structure associated to a bounded geometry metric. When G is uncountable, however, it is not a metric course structure.

7.2. Permanence for groups. Henceforth, all groups are assumed countable and discrete. Further, all properties are assumed to satisfy coarse invariance. Consequently, the statement that a particular group has a particular property is unambiguous.

7.2.1. Theorem. Let $\mathcal{P}$ be a property satisfying subspace and limit permanence. If $\mathrm{G}$ is the direct union of subgroups each having property $\mathcal{P}$ then $\mathrm{G}$ has property $\mathcal{P}$. In particular, a countable discrete group $\mathrm{G}$ satisfies $\mathcal{P}$ if and only if each of its finitely generated subgroups do.

Proof. Fix a proper length function on G. Let $\mathrm{H}$ be the subgroup of $\mathrm{G}$ generated by the elements of the R-ball with center the identity. As this ball is finite it is contained in one of the subgroups comprising the direct union so that the same is true of $\mathrm{H}$. Hence, by subspace permanence $\mathrm{H} \in \mathcal{P}$. Further, the family of cosets $\{\mathrm{sH}\}$, being coarsely equivalent, even isometric to $\mathrm{H}$ in the sense of families, satisfies $\mathcal{P}$. An application of limit permanence based on the subsequent lemma completes the proof.

7.2.2. Lemma. If $\mathrm{G}$ is equipped with a proper length function and the subgroup $\mathrm{H}$ of $\mathrm{G}$ contains the $\mathrm{R}$-ball with center the identity then the cosets of $\mathrm{H}$ are pairwise at distance at least $\mathrm{R}$.

Remark. The above theorem applies, in particular, to the properties $\mathrm{FAD}_{\mathrm{d}}$ and EX. Simple examples, such as the direct sum of countably many copies of $\mathbb{Z}$, show that is does not apply to FAD. 
Remark. The analog for a possibly uncountable group with its canonical coarse structure is treated by Dranishnikov and Smith [13].

We turn to group actions, which we always assume to be by isometries. The simplest situation occurs when a group $\mathrm{G}$ acts metrically properly on a metric space $\mathrm{Y}$. In this case the orbit map

$$
f: G \rightarrow Y, \quad f(s)=s \cdot y
$$

is a coarse embedding; the easiest way to see this is to observe that $\ell(s)=d(y, s \cdot y)$ defines a proper length function on $G$ and to appeal to Proposition 7.1.1. Thus, any property satisfying subspace permanence will pass from $Y$ to $G$. This discussion applies, in particular, when $Y$ is locally finite and the action is free, or has finite stabilizers. We are interested in the complementary case where the action has infinite stabilizers and/or the metric space is not locally finite.

7.2.3. Theorem. Let $\mathcal{P}$ be a property satisfying subspace, finite union and fibering permanence. If $\mathrm{G}$ acts on a locally finite space $\mathrm{Y} \in \mathcal{P}$ and there exists $a \mathrm{y} \in \mathrm{Y}$ for which the stabilizer $\mathrm{G}_{\mathrm{y}} \in \mathcal{P}$ then $\mathrm{G} \in \mathcal{P}$.

Proof. This is a basic application of fibering permanence. Begin by observing that it suffices to consider the case of a transitive action - simply choose $y$ as in the statement and restrict the given action to the orbit $\mathrm{Gy}$ which, as a subspace of $\mathrm{Y}$, satisfies $\mathcal{P}$.

Fix a base point $y \in Y$. We employ the orbit map and begin by checking that it is uniformly expansive. Indeed, this is hinted in the paragraph just before the statement: if $d(s, t) \leq A$ then

$$
d(f(s), f(t))=d\left(y, s^{-1} t \cdot y\right) \leq B=\sup \{d(y, r \cdot y): \ell(r) \leq A\}<\infty .
$$

Apply Lemma 2.1.

It remains to check that the orbit map satisfies the inverse image condition in the statement of fibering permanence. Formally, let $Z \leq Y$ be a bounded family of subspaces of $Y$, say with uniform bound $S$. As we are assuming the action is transitive, we find for every component $Z_{\alpha}$ of $Z$ an element $s_{\alpha} \in G$ for which $s_{\alpha} \cdot Z_{\alpha} \subset B(y, S)$. Using the equivariance of the orbit map we conclude that $f^{-1}(Z)$ is coarsely equivalent (even isometric) as a family, to a family of subspaces of the single metric space

$$
f^{-1}(B(y, S))=\{s \in G: d(y, s \cdot y) \leq S\} .
$$

Thus, by an application of subspace permanence, it remains only to see that this coarse stabilizer satisfies $\mathcal{P}$. But, by local finiteness, it is the finite union of cosets $s G_{y}$, each of which is isometric to $G_{y}$. An application of finite union permanence completes the proof.

7.2.4. Corollary. A property $\mathcal{P}$ satisfying subspace, finite union and fibering permanence is closed under group extensions.

Remark. In the corollary we assume $\mathcal{P}$ satisfies finite union permanence. This is necessary only because we deduce the corollary from the preceding theorem - the corollary remains true without this assumption. Indeed, finite union permanence is used only near the end of the proof to conclude that the union of cosets (7.1) satisfies $\mathcal{P}$. In the case of an extension

$$
1 \rightarrow \mathrm{H} \rightarrow \mathrm{G} \rightarrow \mathrm{G} / \mathrm{H} \rightarrow 1
$$


one verifies directly that this union of cosets is coarsely equivalent to $\mathrm{H}$.

Remark. The proofs of the theorem and corollary apply to the property EX. Brief inspection reveals, however, that the modified form of fibering permanence satisfied by FAD is sufficient, so that the results remain true for FAD, although our approach gives a very poor bound. An analogous result, with the optimal bound, is stated for finitely generated groups in [5, Thm. 7]. The general case is treated in [13, Thm. 2.3]; for an alternate proof see [7, Thm. 5.4]. Compare to the remark just after the statement of Theorem 6.6.

Arguments involving a non-locally finite space follow essentially the same path as the proof of Theorem 7.2.3. The added difficulty occurs near the end of the argument where the infinite union theorem must be applied to show that the coarse stabilizer (7.1) has property $\mathcal{P}$; verification of the excision hypothesis is not always straightforward.

Two cases in which the method has been used effectively are free products (with amalgam) and relative hyperbolicity. In each case, the group under study acts on a (in general) non-locally finite hyperbolic graph - for free products, the Bass-Serre tree and in the case of relative hyperbolicity the 'electric space'. We shall discuss free products below, following a modification of the method of Bell and Dranishnikov [2]. We shall not discuss the case of relative hyperbolicity, but refer to the papers of Osin [19], who developed the method and applied it to FAD, and Dadarlat and Guentner [12], who give a short summary and statements involving the properties EX and CE.

7.2.5. Theorem. A property $\mathcal{P}$ satisfying subspace, union and fibering permanence, and which is possessed by (the vertex set of) a (simplicial) tree is closed under the formation of free products (with amalgam).

Without entering into details we shall describe the proof, following the modification of the original argument of Bell and Dranishnikov given by Dadarlat and Guentner [11, 12]. Willett also gives a very clear discussion in the case of Property $A$ [24]. We hope our informal discussion will complement these resources.

Let $G=A *_{C} B$ be the free product of $A$ and $B$, amalgamated over the common subgroup $C$. We begin by recalling the construction of the Bass-Serre tree of the amalgam. The vertices of the tree are the $A$ and $B$-cosets in $G$, the edges are the C-cosets:

$$
\begin{aligned}
\text { vertices } & =\mathrm{G} / \mathrm{A} \cup \mathrm{G} / \mathrm{B}=\{\mathrm{s} A\} \cup\{\mathrm{tB}\} \\
\text { edges } & =\mathrm{G} / \mathrm{C}=\{\mathrm{rC}\} .
\end{aligned}
$$

Two vertices $v$ and $w$ are connected by an edge precisely when they have non-empty intersection (recall they are subsets of $\mathrm{G}$ ). In this case, one of $v$ and $w$ is an A-coset, the other a B-coset - the intersection $v \cap w$ a $C$-coset which represents the connecting edge. For a proof that this defines a tree, see $[23,1]$. Defining the distance between two vertices to be the length of the shortest edge path connecting them we obtain a metric on (the vertex set of) the tree.

We shall fiber not $G$, but rather a closely related space, over the Bass-Serre tree. Define

$$
X=\left\{x_{v}: x \in v \subset G, v \text { a vertex }\right\}
$$




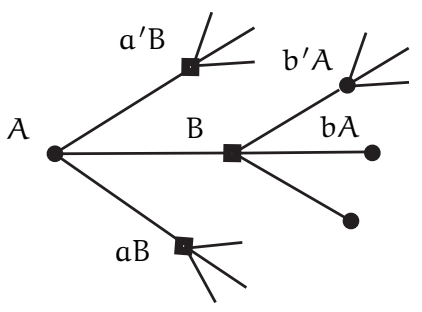

FIGURE 1. Bass-Serre tree.

here $v$ is a vertex in the Bass-Serre tree, viewed as an $A$ or $B$-coset. Thus, $X$ is the disjoint union of the A and B-cosets and, as each element of $\mathrm{G}$ lies in precisely on A-coset and one B-coset, we see that $X$ comprises two (disjoint) copies of G. An adjacency occurs between $x_{v}$ and $x_{w}$, where $x \in \mathrm{G}$ is viewed as an element of two adjacent vertices $v$ and $w$. A transition occurs between $x_{v}$ and $y_{v}$, where $x$ and $y \in G$ are elements of a common $A$ or B-coset $v$.

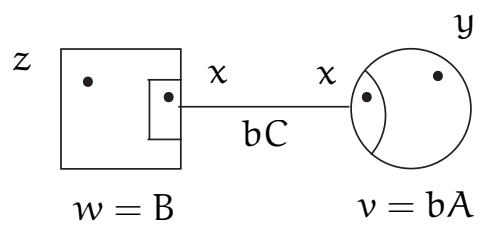

FIGURE 2. Adjacency and transition: $b \in B$.

The metric on $\mathrm{X}$ is the maximal metric satisfying the requirements that an adjacency has distance at most one, and that a transition has distance at most the distance measured in the ambient group $\mathrm{G}$ or, what is the same, in the $\operatorname{coset} v \subset \mathrm{G}$ equipped with the subspace metric. A formula for the metric on $\mathrm{X}$ is given in [11].

Remark. It is important to use the subspace metric on the individual cosets at this stage - this choice eliminates distortion, so that the metric on $\mathrm{X}$ behaves as one would hope. In Figure 2, for example, $d\left(x_{v}, y_{v}\right)$ will actually equal the distance in the ambient group - there is no possibility of finding a shortcut in the neighboring coset $w$. Similarly,

$$
d\left(z_{w}, y_{v}\right)=\inf \{d(y, x)+1+d(x, z): x \in v \cap w=b C\},
$$

where the distances $d(y, x)$ and $d(x, z)$ are measured in the respective cosets $v$ and $w$. This is a special case of the formula from [11]. (In Figure 2 the small rectangle inside the coset $B$ represents the copy of $\mathrm{bC} \subset \mathrm{B}$, and similarly the small semidisk inside the coset $\mathrm{bA}$.)

The group $\mathrm{G}$ acts by isometries on the Bass-Serre tree $\mathrm{T}$ by permuting cosets. Further, $\mathrm{G}$ acts by isometries on $X$ according to the formula $s \cdot x_{v}=(s x)_{s \cdot v}$. And, $f\left(x_{v}\right)=v$ defines an equivariant contraction $X \rightarrow T$ - comparing Figures 1 and 3 the map is evident. We should like to apply 


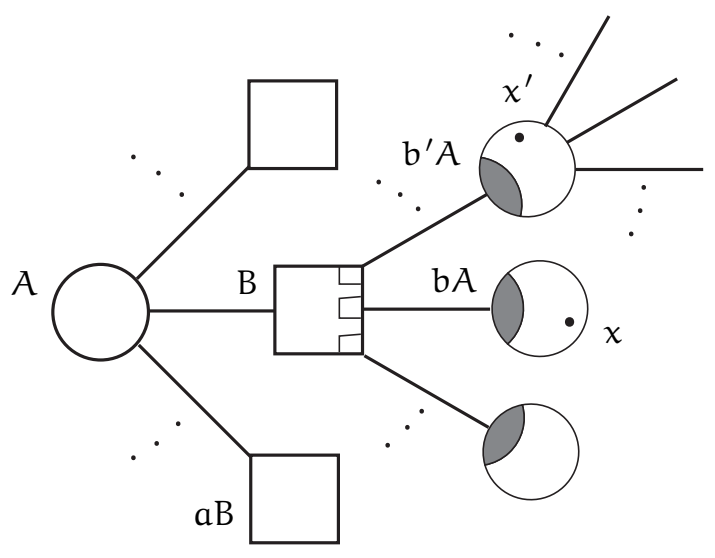

Figure 3. A portion of $X$.

fibering - via a modification of the argument used in the proof of Theorem 7.2.3 - to conclude that $X$ has property $\mathcal{P}$. Since $X$ is locally finite, we would then conclude that $G$ has property $\mathcal{P}$ as well.

The version of fibering we require differs from that in Theorem 7.2.3 in two respects. The first is not substantive - we allow for an equivariant fibering map $X \rightarrow Y$ in place of the orbit map, and assume the action of $\mathrm{G}$ on $\mathrm{Y}$ is cobounded.

7.2.6. Proposition. Let $\mathcal{P}$ be a property satisfying subspace and fibering permanence. Suppose that $\mathrm{G}$ acts on metric spaces $\mathrm{X}$ and $\mathrm{Y}$ by isometries, the action on $\mathrm{Y}$ is cobounded, and that $\mathrm{f}: \mathrm{X} \rightarrow \mathrm{Y}$ is a G-equivariant and uniformly expansive map. Suppose $\mathrm{Y} \in \mathcal{P}$ and that for some $\mathrm{y} \in \mathrm{Y}$ and every $\mathrm{S}$

$$
f^{-1}(B(y, S)) \subset X
$$

satisfies $\mathcal{P}$. Then $\mathrm{X} \in \mathcal{P}$.

The idea, which we encountered in the proof of Theorem 7.2.3, is that the equivariance of $f$ and coboundedness of the action on $\mathrm{Y}$ allow us to reduce the inverse image condition in the statement of fibering permanence to the stated condition on the set $f^{-1}(B(y, S))$.

The second, and more substantive difference concerns the proof that the space appearing in (7.2) satisfies property $\mathcal{P}$. This space is in general an infinite union of cosets, and we must concern ourselves with the hypotheses of infinite union permanence. Fix the basepoint $y=v_{0}=A$. We show by induction on $S$ that

$$
B_{S}=f^{-1}\left(B\left(v_{0}, S\right)\right)=\left\{x_{v} \in X: d_{T}\left(v_{0}, v\right) \leq S\right\}
$$

satisfies $\mathcal{P}$. For $S=0$ we have the coset $v_{0}$, which satisfies $\mathcal{P}$ by hypothesis. Assuming the statement for $S$, we wish to prove it for $S+1$. We write

$$
B_{S+1}=B_{S} \cup \cup\left\{v: d\left(v_{0}, v\right)=S+1\right\}
$$


Excising the R-neighborhood of $B_{S}$ from the spaces comprising this union, the resulting pieces are R-separated. Figure 3 illustrates the passage from $B_{1}$ to $B_{2}$. The shaded region inside the coset $\mathrm{bA}$ represents the R-neighborhood of $\mathrm{bC} \subset \mathrm{bA}$, and similarly for the other shaded regions. The distance $d\left(x, x^{\prime}\right)$ is at least $R-a$ 'path' connecting $x$ and $x^{\prime}$ must lie over the corresponding edge path in $T$, and the first transition from $x$ to $b C$ will already contribute $R$ to the distance. This concludes our discussion of Theorem 7.2.5.

7.2.7. Theorem. Let $\mathcal{P}$ be a property satisfying subspace, union and fibering permanence, and which is possessed by a tree. A group acting cofinitely on a tree (without inversion) ${ }^{6}$ satisfies $\mathcal{P}$ if and only if all vertex stabilizers satisfy $\mathcal{P}$.

Of course, the 'only if' direction is immediate - we are concerned with the 'if' direction. The proof is the same as the one given for free products. Simply fiber over the given tree, carrying out the construction of the total space exactly as before. Both this theorem and Theorem 7.2.5 apply to the properties EX and FAD - again, the modified form of fibering satisfied by FAD is sufficient.

Remark. If $\mathcal{P}$ also satisfies limit permanence we may dispense with the assumption that the action is cofinite. Further, there is an alternate proof. Indeed, assuming the action is without inversion, by the Bass-Serre theory a group as in the statement is built from the vertex stabilizers by repeated free products and HNN-extensions, followed by a limit. Having Theorem 7.2.5 at our disposal, we need only check that an HNN-extension of groups satisfying $\mathcal{P}$ again satisfies $\mathcal{P}$. But, an HNN-extension is itself built from free products, a limit and an extension. See [23, 11].

Remark. Theorem 7.2.5 for FAD, with same estimate inherent here, first appeared in [2]. More refined results for FAD appeared subsequently. A version of Theorem 7.2.7 but with a sharper estimate appeared in [3, Thm. 1] under the assumption of finite generation and a cofinite action. The previous theorem, as applied to FAD, appears to have been overlooked in the literature. Related results may be formulated by replacing the hypothesis of a cofinite action by a uniform bound on the asymptotic dimension of the vertex stabilizers.

\section{COARSE EMBEDDABILITY AND OTHER MISCELLANEA}

As alluded to above, the property of coarse embeddability is somewhat more subtle than the properties of finite asymptotic dimension and exactness. For this reason we are treating CE separately. In addition, we collect several miscellaneous permanence results which do not fit in our general framework.

8.1. Theorem. The property CE satifies coarse invariance, subspace, union and limit permanence.

In this regard, $\mathrm{CE}$ behaves as our other basic properties. An essential difference occurs, however, in relation to fibering permanence - CE satisfies a modified form of fibering permanence in which the base space is assumed to satisfy property EX.

\footnotetext{
${ }^{6}$ This hypothesis is not substantive. Passing to the barycentric subdivision we obtain an action without inversion; the vertex stabilizers for this action are, up to finite index, intersections of vertex stabilizers for the original action.
} 
8.2. Theorem. Let $\mathrm{f}: X \rightarrow y$ be uniformly expansive. Suppose $y \in \mathrm{EX}$ and that for every bounded family of subspaces $z \leq y$ the inverse image $f^{-1}(z) \in \mathrm{CE}$. Then $X \in \mathrm{CE}$.

The failure of the general form of fibering permanence for $\mathrm{CE}$ has interesting consequences in the case of group extensions. Indeed, following the treatment in the previous section, we recover a basic result of Dadarlat and Guentner [11], originally proven directly.

8.3. Theorem. An extension with coarsely embeddable kernel and exact quotient is itself coarsely embeddable. Precisely, if a normal subgroup $\mathrm{H}$ of $\mathrm{G}$ satisfies $\mathrm{CE}$ and $\mathrm{G} / \mathrm{H}$ satisfies $\mathrm{EX}$ then $\mathrm{G}$ satisfies $\mathrm{CE}$.

Apaprt from a couple of essentially obvious remarks, there was little progress on the general problem of extensions until recently. Building on their work regarding stability of a-T-menability under wreath products, deCornulier, Stalder and Valette obtained the following very interesting result [10].

8.4. Theorem. If the countable discrete groups $\mathrm{G}$ and $\mathrm{H}$ are coarsely embeddable then so is their wreath product $\mathrm{H}<\mathrm{G}=\mathrm{H}^{(\mathrm{G})} \rtimes \mathrm{G}$. Here, $\mathrm{H}^{(\mathrm{G})}$ denotes the group of finitely supported $\mathrm{H}$-valued functions on $\mathrm{G}$, on which $\mathrm{G}$ acts by translation.

We shall not enter into the details of the proof, which rests on a characterization of CE in terms of embeddings in spaces with measured walls (basically contained in [21]) and an ingenious construction with such spaces. See [10].

Remark. As is clear from the construction of the wreath product, a property $\mathcal{P}$ satisfying our primitive permanence results will be closed under formation of wreath products. Indeed, it follows from fibering permanence that finite sums of $H$ satisfy $\mathcal{P}$, from limit permanence that $\mathrm{H}^{(\mathrm{G})}$ satisfies $\mathcal{P}$, and from another application of fibering permanence (an extension) that $\mathrm{H} \imath \mathrm{G}$ satisfies $\mathcal{P}$. Thus, property EX is closed under formation of wreath products and, in keeping with the above, if $H \in C E$ and $G \in E X$ then $H$ $\& \in C E$. Thus, the content in the theorem is in the weakened hypothesis on $\mathrm{G}$.

We conclude our discussion of CE with remarks concerning limits, coarse spaces, and a caveat. A countable discrete group satisfies CE precisely when its finitely generated subgroups do. This follows from Theorems 8.1 and 7.2.1; see also [11] for the original proof. Generalized limit permanence results valid in the setting of coarse spaces would be applicable to non-necessarily countable groups. In the case of $\mathrm{FAD}_{\mathrm{d}}$ a generalized form of limit permanence was obtained by Dranishnikov and Smith $[13,4]: G \in F A D_{d}$ precisely when each of its finitely generated subgroups satisfies $F A D_{d}$. But, the generalization fails for property $C E$. An example is $G=\mathbb{R}$. Equip $G$ with the canonical coarse structure described earlier. As this coarse structure is not metrizable, $G$ cannot satisfy CE. Nevertheless, the finitely generated generated subgroup of G are (up to isomorphism) precisely the groups $\mathbb{Z}^{n}$, for some $n$ and each of these satisfies $C E$.

Finally, we complete our collection of miscellaneous remarks by mentioning work of Ozawa, which provides an alternate approach to the permanence of Property A with respect to (amalgamated) free products, and relative hyperbolicity [20]. 
8.5. Theorem. Suppose G acts on a countable, fine hyperbolic graph with finite quotient. If all vertex stabilizers satisfy EX then so does $\mathrm{G}$.

We refer to the original paper for definitions of the terms appearing in the statement. Suffice it to say that a tree is a fine hyperbolic graph, and that the theorem applies to free products with arbitrary amalgam. At the heart of the proof is an elegant piece of functional analysis - an application of the Hahn-Banach theorem. The method appears a bit sharper than the more geometric method introduced by Bell and Dranishnikov which we have followed in that it produces a compact amenable G-space. See the paper of Ozawa for an application of this idea [20].

Finally, the method of proof of Theorem 8.5 can be extended to prove that certain Artin groups satisfy EX. These groups, at least at present, cannot be treated using the geometric approach. See [16].

\section{REFERENCES}

[1] G. Baumslag. Topics in Combinatorial Group Theory. ETH Lectures in Mathematics. Birkhäuser, Boston, 1993.

[2] G. Bell and A. Dranishnikov. On asymptotic dimension of groups. Algebr. Geom. Topol., 1:57-71 (electronic), 2001.

[3] G. Bell and A. Dranishnikov. On asymptotic dimension of groups acting on trees. Geom. Dedicata, 103:89-101, 2004.

[4] G. Bell and A. Dranishnikov. Asymptotic dimension. Topology Appl., 155(12):1265-1296, 2008.

[5] G. C. Bell and A. N. Dranishnikov. A Hurewicz-type theorem for asymptotic dimension and applications to geometric group theory. Trans. Amer. Math. Soc., 358(11):4749-4764 (electronic), 2006.

[6] Gregory C. Bell. Property A for groups acting on metric spaces. Topology Appl., 130(3):239-251, 2003.

[7] N. Brodskiy, J. Dydak, M. Levin, and A. Mitra. A Hurewicz theorem for the Assouad-Nagata dimension. J. Lond. Math. Soc. (2), 77(3):741-756, 2008.

[8] J. Brodzki, G. A. Niblo, and N. J. Wright. Property A, partial translation structures, and uniform embeddings in groups. J. Lond. Math. Soc. (2), 76(2):479-497, 2007.

[9] Xiaoman Chen, Romain Tessera, Xianjin Wang, and Guoliang Yu. Metric sparsification and operator norm localization. Adv. Math., 218(5):1496-1511, 2008.

[10] Y. Cornulier, Y. Stalder, and A. Valette. Proper actions of wreath products and generalizations. arXiv:0905.3960, 2009.

[11] Marius Dadarlat and Erik Guentner. Constructions preserving Hilbert space uniform embeddability of discrete groups. Trans. Amer. Math. Soc., 355(8):3253-3275 (electronic), 2003.

[12] Marius Dadarlat and Erik Guentner. Uniform embeddability of relatively hyperbolic groups. J. Reine Angew. Math., 612:1-15, 2007.

[13] A. Dranishnikov and J. Smith. Asymptotic dimension of discrete groups. Fund. Math., 189(1):27-34, 2006.

[14] A. N. Dranishnikov, G. Gong, V. Lafforgue, and G. Yu. Uniform embeddings into Hilbert space and a question of Gromov. Canad. Math. Bull., 45(1):60-70, 2002.

[15] M. Gromov. Asymptotic invariants of infinite groups. In A. Niblo and M. Roller, editors, Geometric Group Theory, number 182 in London Mathematical Society Lecture Notes, pages 1-295. Cambridge University Press, 1993.

[16] E. Guentner and G. Niblo. Complexes and exactness of certain Artin groups. In preparation, 2010.

[17] E. Guentner, R. Tessera, and G. Yu. Decomposition complexity and the stable Borel conjecture. Preprint, 2010.

[18] Piotr W. Nowak. Coarsely embeddable metric spaces without Property A. J. Funct. Anal., 252(1):126-136, 2007.

[19] D. Osin. Asymptotic dimension of relatively hyperbolic groups. Int. Math. Res. Not., 35:2143-2161, 2005. 
[20] Narutaka Ozawa. Boundary amenability of relatively hyperbolic groups. Topology Appl., 153(14):2624-2630, 2006.

[21] Guyan Robertson and Tim Steger. Negative definite kernels and a dynamical characterization of property (T) for countable groups. Ergodic Theory Dynam. Systems, 18(1):247-253, 1998.

[22] J. Roe. Lectures on Coarse Geometry, volume 31 of University Lecture Series. American Mathematical Society, Providence, RI, 2003.

[23] J. P. Serre. Trees. Springer, New York, 1980. Translation from French of "Arbres, Amalgames, SL $L_{2}$ ", Astérisque no. 46.

[24] Rufus Willett. Some notes on property A. In Limits of graphs in group theory and computer science, pages 191-281. EPFL Press, Lausanne, 2009.

[25] G. Yu. The Coarse Baum-Connes conjecture for spaces which admit a uniform embedding into Hilbert space. Inventiones Math., 139:201-240, 2000.

University of Hawai 'i at Mānoa, Department of Mathematics, 2565 McCarthy Mall, HonOLULU, HI 96822

E-mail address: erik@math.hawaii.edu 